\title{
Cigarette and Waterpipe Smoking are Associated with the Risk of Stroke in Lebanon
}

\author{
Maya El-Hajj ${ }^{1,2,}$, Pascale Salameh ${ }^{1}$, Samar Rachidi ${ }^{1}$, Amal Al-Hajje ${ }^{1}$, Hassan Hosseini ${ }^{2,3}$ \\ ${ }^{1}$ Clinical and Epidemiological Research Laboratory, Faculty of Pharmacy, Lebanese University, Hadath, Lebanon \\ ${ }^{2}$ Department of Neurology, UMR 955, IMRM, Institut Mondor de Recherche Biomédicale, Université Paris-Est, Créteil, France \\ ${ }^{3}$ Department of Neurology, Hôpital Henri-Mondor, UPEC, Assistance Publique-Hôpitaux de Paris (AP-HP), Créteil, France
}

ARTICLE INF O
Article History
Received 10 December 2017
Accepted 5 April 2018
Keywords
Smoking
stroke
Lebanon
case-control study
epidemiology

\begin{abstract}
Cigarette and waterpipe (shisha and hookah) smoking are main public health concerns in Lebanon. We aim to assess the relationship between smoking and stroke, mainly waterpipe smoking, to better apply preventive and therapeutic interventions. A case-control study was conducted at five tertiary private and governmental hospitals in Lebanon between January 1st, 2015 and December 31st, 2016, using a standardized questionnaire. A stepwise ascending logistic regression was conducted. Odds ratio through $95 \%$ confidence interval (CI) expressed the degree of association among variables. In total, 650 patients were involved in the study with 205 stroke cases and 445 stroke-free normal individuals considered as controls. The risk of stroke was found to increase significantly among current cigarette smokers; total stroke Odds ratio (OR) $=2.79$ (95\% CI, 1.72-4.54), ischemic stroke $\mathrm{OR}=2.59$ (95\% CI, 1.46-4.59), and hemorrhagic stroke OR $=4.25$ (95\% CI, 1.33-13.59). The risk of total and ischemic stroke was also found to increase significantly among current waterpipe smokers; OR 4.99 (95\% CI, 2.07-11.99) and 6.25 (95\% CI, 2.26-17.39), respectively. Moreover, waterpipe smoking was observed to have a stronger association with stroke in current cigarette smokers than non-smokers. Waterpipe smoking was found to be associated with stroke in this study. Moreover, this association is stronger among cigarette smokers, demonstrating a quantitative interaction between waterpipe and cigarette smoking.
\end{abstract}

(C) 2019 Atlantis Press International B.V.

This is an open access article distributed under the CC BY-NC 4.0 license (http://creativecommons.org/licenses/by-nc/4.0/).

\section{INTRODUCTION}

Cigarette smoking is an important risk factor for stroke [1,2]. It is the main public health concern in Lebanon, where it has been associated with a substantial number of diseases including cardiovascular disease, cancer, hypertension, diabetes, and dyslipidemia. According to the World Health Organization (WHO), in 2015, the age-standardized prevalence of cigarette smoking varied between $37.0 \%$ and $24.2 \%$ and of any smoked tobacco between $43.9 \%$ and $29.9 \%$, for males and females, respectively, among individuals aged 15 years or older [3].

The prevalence of waterpipe smoking (shisha and hookah) has reached $20-30 \%$ of adults during the past few years [4]. In Lebanon, the prevalence of waterpipe smoking has increased extensively, reaching 15\% among Lebanese adults in general, and 35.9-51.9\% among males and females 40 years of age or older, respectively $[5,6]$. About $36.5 \%$ of exclusive Lebanese waterpipe smokers smoke more than seven waterpipes per week [7]. Lebanon is considered the first country in comparison with other countries in regard to current waterpipe smoking among school students (25\%) and is the second after Pakistan among university students (28\%) [5]. In addition, about 5-6\% of Lebanese expectant mothers were reported to smoke waterpipe during pregnancy [5].

\footnotetext{
*Corresponding author.Email: hajj_maya@hotmail.com
}

As a result of the smoking situation in Lebanon, especially waterpipe smoking and its high popularity among Lebanese youth, this study aims to assess the relationship between smoking and stroke, mainly waterpipe smoking, to better apply preventive and therapeutic interventions.

\section{MATERIALS AND METHODS}

\subsection{Study Design}

A case-control study was conducted at five tertiary private and governmental hospitals in Lebanon between January 1st, 2015 and December 31st, 2016. This study was observational with guaranteed anonymity of patients, therefore, the ethical committee of the Lebanese University (Faculty of Pharmacy) forwent the study approval with respect to the ethical values and rules [8]. Patients included in the study provided an oral consent.

\subsection{Questionnaire for Data Collection}

The questionnaire was divided into four parts, including (1) sociodemographic factors and diagnosis and drug exposure, (2) patients' symptoms, (3) potential risk factors of stroke including smoking, and (4) laboratory data. 
The questionnaire, administered in Arabic to patients, included the following information in relation to smoking:

(a) Current cigarette smoking: period and number of cigarettes smoked.

(b) Former cigarette smoking: period of smoking, number of cigarettes previously smoked, and period of smoking cessation.

(c) Passive cigarette smoking: close family living with patient and smoking cigarettes, work/community, and number of cigarettes exposed to by the patient.

(d) Current waterpipe smoking: period of smoking and number of waterpipes smoked.

(e) Former waterpipe smoking: period of smoking, number of waterpipes previously smoked, and period of smoking cessation.

(f) Passive waterpipe smoking: close family living with the patient and smoking waterpipes, work/community, and number of waterpipes exposed to by the patient.

\subsection{Data Collection and Definition of Stroke}

Data were collected contemporarily after patients' diagnosis from hospital files and/or directly from patients/proxy (a proxy was considered as an authorized person, closely living with the patient and who knew everything about the patient's medical history). A proxy was only interviewed when the patient was incapable of answering the questions. All the data regarding sociodemographic factors, diagnosis, medical history, and laboratory tests were collected from patients' hospitals files.

\subsubsection{Stroke}

Stroke is defined by the WHO as "rapidly developing clinical signs of focal (or global) disturbance of cerebral function, with symptoms lasting $24 \mathrm{~h}$ or longer or leading to death, with no apparent cause other than vascular origin" [9].

The diagnosis of stroke and subtypes was confirmed from the physician's diagnosis and imaging as recorded in the patient's file [10].

\subsection{Inclusion and Exclusion Criteria}

Inclusion of cases required being Lebanese, hospitalized, $\geq 18$ years old, and diagnosed with stroke or transient ischemic attack (TIA) using brain computed tomography (CT) and/or magnetic resonance imaging (MRI). Exclusion of cases relied on unavailability of oral consent, clinical information, or CT/MRI report.

Inclusion of controls required being Lebanese, $\geq 18$ years old, and hospitalized at the same hospitals as cases for diseases other than stroke or TIA. Controls were admitted to hospitals for infection, cancer, injuries, surgeries, fractures, as well as kidney, endocrinology, and digestive diseases, and were excluded if they did not provide an oral consent to conduct the survey.

\subsection{Sample Size Calculation}

A recently published study in Lebanon about stroke risk factors reported Odds ratio $(\mathrm{OR})=2.02(95 \% \mathrm{CI}, 1.19-3.44)$ for cigarette smokers [11]. According to WHO, the prevalence of cigarette smoking in Lebanon is about $30.7 \%$ for Lebanese 15 years of age or older [5]. We set the two-sided confidence level at $95 \%$ and the power at $80 \%$, and considered two controls for each stroke case. A total of $300 \mathrm{sub}-$ jects was required for this study with 100 cases and 200 controls [11].

\subsection{Statistical Methods}

SPSS version 24 (IBM Corp., released 2016, Windows version, Armonk, NY) was used for the analysis, with $p$-value $<0.05$ determining statistical significance. Means with standard deviation represented continuous variables and percentages represented categorical variables. Chi-square test, Fisher's Exact test, and Student's $t$-test were used to differentiate among baseline characteristics between cases and controls.

Stepwise ascending logistic regression was conducted to eliminate the effect of confounding. The following factors were included in the multivariate analysis: socioeconomic and demographic factors, medication, medical history, and behavioral history (including smoking). Patients followed specific criteria to be classified as obese or not, and physically active or not $[12,13]$. All variables were included in the multivariate analysis, even with $p$-value $>0.2$ in the bivariate (employment and insurance), because many published studies considered these factors to be associated with stroke.

Collinearity between independent variables was conducted prior to logistic regression. In case of significant collinearity existing between two variables (i.e., $r>0.7$ ), only one variable was considered in the analysis.

To calculate the cumulative dosing of cigarette/waterpipes smoking, smoking duration (in years) was multiplied by the mean number of daily cigarette packs or number of times waterpipes were used weekly. Passive smoking was assessed based on the cumulative dosing of cigarette/waterpipe smoke the non-smoker was exposed to from family members, work, and/or community during the day.

Odds ratio through 95\% confidence interval expressed the degree of association between the independent variables and the dependent variable (presence of stroke or not).

Stratification analysis was also conducted to measure the association among waterpipe smoking and stroke in cigarette and noncigarette smokers.

\section{RESULTS}

In total, 650 patients were involved in the study of which 205 were stroke cases and 445 stroke-free normal controls. Imaging/clinical information were missing for 12 stroke patients, and therefore were excluded from the study.

Among included subjects, $34.6 \%$ and $1.3 \%$ of stroke cases and controls, respectively, had a history of stroke. Among the 205 included stroke cases, $69.8 \%, 15.1 \%$, and $15.1 \%$ were ischemic, hemorrhagic, 
and TIA, respectively. Imaging were conducted for all included cases: $75.1 \%$ (CT scan), 16.1\% (MRI), and 8.8\% (CT and MRI).

Around $81.8 \%$ of study participants were from Beirut and Mount Lebanon. A one-way ANOVA test indicated no differences between included hospitals $(p=0.135)$.

\subsection{Socioeconomic and Demographic Differences Between Stroke and Stroke-free Patients}

The mean age of stroke cases was about $70 \pm 12$ years. Included stroke patients were mainly female, unemployed, married, medically insured, and had a low education level. Age ( $\geq 65$ years), education status, and marital status statistically differed between cases and controls (Table 1).

\subsection{Medical and Health Characteristics of the Study Sample}

Stroke patients and stroke-free persons statistically differed in regard to medication history, body mass index, medical history, and behavioral history as well as systolic and diastolic blood pressures (Table 2) [14].

\subsection{Assessment of cigarette and waterpipe smoking as stroke risk factors}

\subsubsection{Participants' smoking characteristics}

Stroke patients statistically differed from stroke-free persons in regard to being a current cigarette smoker and a current and former waterpipe smoker and being exposed to secondhand smoking at home (among non-smokers) (Table 3 ).

\subsubsection{Relationship between smoking and stroke}

All sociodemographic, vascular, and behavioral factors (physical activity) were controlled in the multivariate analysis. Being a current cigarette smoker statistically increased the risk of total stroke OR $=2.79(95 \% \mathrm{CI}, 1.72-4.54)$, ischemic stroke OR = 2.59 (95\% CI, 1.46-4.59), and hemorrhagic stroke OR $=4.25$

Table 1 Data on socioeconomic and demographic characteristics of participants

\begin{tabular}{|c|c|c|c|c|}
\hline Variables & Total $(N=650)$ & Stroke patients $(N=205)$ & Stroke-free patients $(N=445)$ & $p$-Value \\
\hline Age (years), mean \pm SD & $63.3 \pm 16.5$ & $69.6 \pm 12.01$ & $60.4 \pm 17.4$ & $<0.001^{\star}$ \\
\hline Age group (years), $N(\%)$ & & & & $<0.001^{\star}$ \\
\hline $18-44$ & $96(14.8)$ & $6(2.9)$ & $90(20.2)$ & \\
\hline $45-49$ & $34(5.2)$ & $6(2.9)$ & $28(6.3)$ & \\
\hline $50-54$ & $56(8.6)$ & $13(6.3)$ & $43(9.7)$ & \\
\hline $55-59$ & $42(6.5)$ & $11(5.4)$ & $31(7.0)$ & \\
\hline $60-64$ & $45(6.9)$ & $14(6.8)$ & $31(7.0)$ & \\
\hline $65-69$ & $102(15.7)$ & $45(22.0)$ & $57(12.8)$ & \\
\hline $70-74$ & $79(12.2)$ & $37(18.0)$ & $42(9.4)$ & \\
\hline $75-79$ & $93(14.3)$ & $32(12.2)$ & $61(13.7)$ & \\
\hline $80-84$ & $74(11.4)$ & $25(12.2)$ & $49(11.0)$ & \\
\hline$\geq 85$ & $29(4.5)$ & $16(7.8)$ & $13(2.9)$ & \\
\hline Sex, $N(\%)$ & & & & 0.169 \\
\hline Male & $265(40.8)$ & $92(44.9)$ & $173(38.9)$ & \\
\hline Female & $385(59.2)$ & $113(55.1)$ & $272(61.1)$ & \\
\hline Employment status, $N(\%)$ & & & & 0.674 \\
\hline Unemployed & $413(63.6)$ & $130(63.2)$ & $284(63.7)$ & \\
\hline Retired & $89(13.6)$ & $31(15.2)$ & $57(12.8)$ & \\
\hline Employed & $148(22.8)$ & $44(21.6)$ & $104(23.4)$ & \\
\hline Education status, $N(\%)$ & & & & $0.001^{\star}$ \\
\hline Low education & $498(76.7)$ & $175(85.4)$ & $323(72.7)$ & \\
\hline Intermediate & $85(13.0)$ & $21(10.2)$ & $64(14.2)$ & \\
\hline Secondary & $11(1.7)$ & $3(1.5)$ & $8(1.8)$ & \\
\hline Higher education & $56(8.6)$ & $6(2.9)$ & $50(11.3)$ & \\
\hline Marital status, $N(\%)$ & & & & $0.002^{\star}$ \\
\hline Single & $39(6.0)$ & $6(2.9)$ & $33(7.4)$ & \\
\hline Married & $451(69.4)$ & $133(64.9)$ & $318(71.5)$ & \\
\hline Widowed/divorced & $160(24.6)$ & $66(32.2)$ & $94(21.1)$ & \\
\hline Monthly income (L.L.), $N(\%)$ & & & & 0.050 \\
\hline$<500,000$ & $359(56.4)$ & $129(64.5)$ & $230(52.8)$ & \\
\hline $500,000-1,000,000$ & $207(32.5)$ & $54(27.0)$ & $153(35.1)$ & \\
\hline $1,000,000-2,000,000$ & $65(10.2)$ & $16(8.0)$ & $49(11.2)$ & \\
\hline$>2,000,000$ & $5(0.8)$ & $1(0.5)$ & $4(0.9)$ & \\
\hline Medical insurance, $N(\%)$ & & & & 0.489 \\
\hline Insured & $397(61.1)$ & $121(59.0)$ & $276(62.0)$ & \\
\hline Uninsured & $253(38.9)$ & $84(41)$ & $169(38.0)$ & \\
\hline
\end{tabular}

${ }^{*} p<0.05$, statistically significant; L.L., Lebanese Lira; SD, standard deviation; TIA/CVA, transient ischemic attack/cerebrovascular accident. 
Table 2 Medical and health characteristics of the study sample

\begin{tabular}{|c|c|c|c|c|c|c|}
\hline \multirow[b]{2}{*}{ Variables } & \multirow[b]{2}{*}{$\begin{array}{c}\text { Total } \\
(N=650)\end{array}$} & \multicolumn{3}{|c|}{ Stroke patients $(N=205)$} & \multirow{2}{*}{$\begin{array}{c}\text { Stroke-free } \\
\text { individuals } \\
(N=445)\end{array}$} & \multirow[b]{2}{*}{$p$-Value } \\
\hline & & $\begin{array}{c}\text { TIA } \\
(N=31)\end{array}$ & $\begin{array}{l}\text { Ischemic } \\
(N=143)\end{array}$ & $\begin{array}{l}\text { Hemorrhagic } \\
\qquad(N=31)\end{array}$ & & \\
\hline \multirow[t]{2}{*}{ Body mass index $\left(\mathrm{Kg} / \mathrm{m}^{2} ;\right.$ mean $\left.\pm \mathrm{SD}\right)$} & \multirow[t]{2}{*}{$26.5 \pm 5.1$} & \multicolumn{3}{|c|}{$27.2 \pm 5.4$} & \multirow[t]{2}{*}{$26.2 \pm 4.9$} & \multirow[t]{2}{*}{$0.016^{\star}$} \\
\hline & & $28.9 \pm 6.2$ & $27 \pm 4.7$ & $26.4 \pm 7.1$ & & \\
\hline \multicolumn{7}{|l|}{ Medication history $N(\%)$} \\
\hline \multirow[t]{2}{*}{ Antihypertensive } & \multirow[t]{2}{*}{$274(42.2)$} & & $140(68.3)$ & & \multirow[t]{2}{*}{$134(30.1)$} & \multirow[t]{2}{*}{$<0.001^{\star}$} \\
\hline & & $16(51.6)$ & $96(67.1)$ & $28(90.3)$ & & \\
\hline Lipid lowering medication & $153(23.5)$ & & $70(34.1)$ & & $83(18.7)$ & $<0.001^{*}$ \\
\hline & & $13(41.9)$ & $50(35)$ & $7(22.6)$ & & \\
\hline Antidiabetes & $174(26.8)$ & & $72(35.1)$ & & $102(22.9)$ & $0.002^{\star}$ \\
\hline & & $14(45.2)$ & $49(34.3)$ & $9(29)$ & & \\
\hline Antidepressant & $86(13.2)$ & & $36(17.6)$ & & $50(11.2)$ & $0.034^{\star}$ \\
\hline & & $3(9.7)$ & $30(21)$ & $3(9.7)$ & & \\
\hline Anticoagulant & $47(7.2)$ & & $29(14.1)$ & & $18(4.0)$ & $<0.001^{\star}$ \\
\hline & & $10(32.3)$ & $16(11.2)$ & $3(9.7)$ & & \\
\hline Aspirin & $210(32.3)$ & & $99(48.3)$ & & $111(24.9)$ & $<0.001^{\star}$ \\
\hline & & $10(32.3)$ & $77(53.8)$ & $12(38.7)$ & & \\
\hline Clopidogrel & $60(9.2)$ & & $29(14.1)$ & & $31(7.0)$ & $0.005^{\star}$ \\
\hline & & $3(9.7)$ & $25(17.5)$ & $1(3.2)$ & & \\
\hline Medical history $N(\%)$ & & & & & & \\
\hline Hypertension & $278(42.8)$ & & $152(74.1)$ & & $126(28.3)$ & $<0.001^{\star}$ \\
\hline & & $16(51.6)$ & $108(75.5)$ & $28(90.3)$ & & \\
\hline Diabetes mellitus & $172(26.5)$ & & $75(36.6)$ & & $97(21.8)$ & $<0.001^{*}$ \\
\hline & & $14(45.2)$ & $51(35.7)$ & $10(32.3)$ & & \\
\hline Dyslipidemia & $137(21.1)$ & & $62(30.2)$ & & $75(16.9)$ & $<0.001^{\star}$ \\
\hline & & $12(38.7)$ & $44(30.8)$ & $6(19.4)$ & & \\
\hline Cardiac arrhythmia/Atrial fibrillation & $93(14.3)$ & & $57(27.8)$ & & $36(8.1)$ & $<0.001^{\star}$ \\
\hline & & $3(9.7)$ & $45(31.5)$ & $9(29)$ & & \\
\hline Coronary heart disease/Myocardial infarction & $128(16.2)$ & & $87(42.4)$ & & $41(9.2)$ & $<0.001^{*}$ \\
\hline & & $9(29)$ & $64(44.8)$ & $14(45.2)$ & & \\
\hline Peripheral artery disease & $24(3.7)$ & & $17(8.3)$ & & $7(1.6)$ & $<0.001^{\star}$ \\
\hline & & $0(0)$ & $14(9.8)$ & $3(9.7)$ & & \\
\hline Heart failure & $65(10.0)$ & & $35(17.1)$ & & $30(6.7)$ & $<0.001^{\star}$ \\
\hline & & $6(19.4)$ & $26(18.2)$ & $3(9.7)$ & & \\
\hline Deep venous thrombosis/Pulmonary embolism & $41(6.3)$ & & $25(12.2)$ & & $16(3.6)$ & $<0.001^{*}$ \\
\hline & & $5(16.1)$ & $19(13.3)$ & $1(3.2)$ & & \\
\hline Obesity & $144(22.3)$ & & $53(25.9)$ & & $91(20.6)$ & 0.128 \\
\hline & & $12(38.7)$ & $33(23.4)$ & $8(25.8)$ & & \\
\hline Physically active & $251(38.6)$ & & $58(28.3)$ & & $193(43.4)$ & $<0.001^{*}$ \\
\hline & & $12(38.7)$ & $42(29.4)$ & $4(12.9)$ & & \\
\hline Cigarette smoking status $N(\%)$ & & & & & & $<0.001^{*}$ \\
\hline Never smokers & $410(63.1)$ & & $106(51.7)$ & & $304(68.3)$ & \\
\hline & & $13(41.9)$ & $82(57.3)$ & $11(35.5)$ & & \\
\hline Current smokers & $194(29.8)$ & & $83(40.5)$ & & $111(24.9)$ & \\
\hline & & $12(38.7)$ & $52(36.4)$ & $19(61.3)$ & & \\
\hline Former smokers & $46(7.1)$ & & $16(7.8)$ & & $30(6.7)$ & \\
\hline & & $6(19.4)$ & $9(6.3)$ & $1(3.2)$ & & \\
\hline Waterpipe smoking status $N(\%)$ & & & & & & $0.001^{\star}$ \\
\hline Never smokers & $584(89.8)$ & & $172(83.9)$ & & $412(92.6)$ & \\
\hline & & $23(74.2)$ & $121(84.6)$ & $28(90.3)$ & & \\
\hline Current smokers & $47(7.2)$ & & $21(10.2)$ & & $26(5.8)$ & \\
\hline & & $2(6.5)$ & $16(11.2)$ & $3(9.7)$ & & \\
\hline Former smokers & $19(2.9)$ & & $12(5.9)$ & & $7(1.6)$ & \\
\hline & & $6(19.4)$ & $6(4.2)$ & $0(0)$ & & \\
\hline Blood pressure classification on admission $N(\%)$ & & & & & & $<0.001^{\star}$ \\
\hline Grade 1 & $95(14.6)$ & & $62(30.2)$ & & $33(7.4)$ & \\
\hline & & $9(29)$ & $51(35.7)$ & $2(6.5)$ & & \\
\hline Grade 2 & $39(6.0)$ & & $34(16.6)$ & & $5(1.1)$ & \\
\hline & & $1(3.2)$ & $19(13.3)$ & $14(45.2)$ & & \\
\hline Grade 3 & $24(3.7)$ & & $22(10.7)$ & & $2(0.4)$ & \\
\hline & & $3(9.7)$ & $12(8.4)$ & $7(22.6)$ & & \\
\hline
\end{tabular}

*Statistically significant; The World Health Organization-International Society of Hypertension classifies blood pressure (BP) according to three grades: grade 1, systolic blood pressure (SBP) 140-159 mmHg and diastolic blood pressure (DBP) 90-99 mmHg; grade 2, SBP 160-179 $\mathrm{mmHg}$ and DBP 100-109 mmHg; grade 3, SBP $\geq 180 \mathrm{mmHg}$ and DBP $\geq 110 \mathrm{mmHg}$ [14]. 
Table 3 Participants' smoking characteristics

\begin{tabular}{|c|c|c|c|c|c|}
\hline Variables & Total $(N=650)$ & $\begin{array}{l}\text { Stroke patients } \\
\quad(N=205)\end{array}$ & $\begin{array}{l}\text { Stroke-free individuals } \\
\qquad(N=445)\end{array}$ & OR (95\% CI) & $p$-Value \\
\hline \multicolumn{6}{|c|}{ Cigarette smoker vs. never smoker } \\
\hline Never smoker & $410(63.1)$ & $106(51.7)$ & $304(68.3)$ & 1.00 & \\
\hline Current smoker & $194(29.8)$ & $83(40.5)$ & $111(24.9)$ & $2.14(1.49-3.07)$ & $<0.001^{*}$ \\
\hline Former smoker & $46(7.1)$ & $16(7.8)$ & $30(6.7)$ & $1.53(0.80-2.92)$ & 0.197 \\
\hline \multicolumn{6}{|c|}{ Waterpipe smoker vs. never smoker } \\
\hline Never smoker & $584(89.9)$ & $172(83.9)$ & $412(92.6)$ & 1.00 & \\
\hline Current smoker & $47(7.2)$ & $21(10.2)$ & $26(5.8)$ & $1.94(1.06-3.53)$ & $0.032^{*}$ \\
\hline Former smoker & $19(2.9)$ & $12(5.9)$ & $7(1.6)$ & $4.11(1.59-10.61)$ & $0.004^{*}$ \\
\hline \multicolumn{6}{|c|}{$\begin{array}{l}\text { Passive cigarette smoking at home vs. never smoker with } \\
\text { no passive smoking }\end{array}$} \\
\hline Yes & $167(25.7)$ & $63(30.7)$ & $104(23.4)$ & $1.46(1.01-2.10)$ & $0.047^{*}$ \\
\hline \multicolumn{6}{|c|}{$\begin{array}{l}\text { Passive cigarette smoking at work/community vs. never } \\
\text { smoker with no passive smoking }\end{array}$} \\
\hline Yes & $27(4.2)$ & $12(5.9)$ & $15(3.4)$ & $1.78(0.82-3.88)$ & 0.145 \\
\hline
\end{tabular}

*Statistically significant; CI, confidence interval; OR, odds ratio.

Table 4 Logistic regression: relationship between smoking and stroke

\begin{tabular}{|c|c|c|c|}
\hline \multirow{2}{*}{ Major independent variables } & \multirow{2}{*}{$\begin{array}{c}\text { OR adjusted }(95 \% \mathrm{CI}), p \\
\text { Total stroke }(N=205)\end{array}$} & \multirow{2}{*}{$\begin{array}{l}\text { OR adjusted }(95 \% \mathrm{CI}), p \\
\text { Ischemic stroke }(N=143)\end{array}$} & \multirow{2}{*}{$\begin{array}{c}\text { OR adjusted }(95 \% \mathrm{CI}), p \\
\text { Hemorrhagic stroke }(N=31)\end{array}$} \\
\hline & & & \\
\hline Current cigarette smoking & $2.79(1.72-4.54),<0.001^{\star}$ & $2.59(1.46-4.59), 0.001^{\star}$ & $4.25(1.33-13.59), 0.015^{\star}$ \\
\hline Current waterpipe smoking & $4.99(2.07-11.99),<0.001^{*}$ & $6.26(2.26-17.39),<0.001^{*}$ & $3.16(0.32-31.43), 0.327$ \\
\hline Age ( $\geq 65$ years) & $1.03(1.01-1.05), 0.006^{*}$ & $1.04(1.02-1.07), 0.001^{\star}$ & $0.98(0.92-1.04), 0.438$ \\
\hline Sex (female) & $1.16(0.67-2.02), 0.603$ & $1.31(0.68-2.56), 0.419$ & $0.23(0.03-1.75), 0.154$ \\
\hline Hypertension history (presence) & $12.56(3.77-41.82),<0.001^{*}$ & $14.24(4.02-50.47),<0.001^{*}$ & 37.09 (0.7-1964.71), 0.074 \\
\hline Diabetes history (presence) & $1.26(0.4-4.0), 0.696$ & $1.51(0.38-6.02), 0.556$ & $0.38(0.03-5.53), 0.480$ \\
\hline Dyslipidemia history (presence) & $0.76(0.35-1.64), 0.481$ & $0.88(0.39-2.02), 0.77$ & $0.36(0.06-2.23), 0.273$ \\
\hline Cardiac diseases history (presence) & $1.87(1.08-3.26), 0.026^{*}$ & $2.28(1.22-4.24), 0.009^{*}$ & $4.96(1.1-22.47), 0.038^{*}$ \\
\hline Overweight and obesity history (presence) & $2.28(1.53-3.39),<0.001^{\star}$ & $2.36(1.5-3.72),<0.001^{\star}$ & $9.28(2.93-29.37),<0.001^{\star}$ \\
\hline Deep venous thrombosis/pulmonary embolism history (presence) & $1.96(0.74-5.18), 0.175$ & $2.36(0.84-6.61), 0.104$ &,- 0.998 \\
\hline Physical activity (physically active vs. physically inactive) & $0.46(0.29-0.73), 0.001^{*}$ & $0.51(0.29-0.87), 0.013^{*}$ & $0.012(0.03-0.48), 0.003^{*}$ \\
\hline Taking antihypertensive & $0.25(0.08-0.87), 0.029^{*}$ & $0.2(0.05-0.71), 0.013^{\star}$ & $1.53(0.03-72.29), 0.828$ \\
\hline Taking lipid lowering medication & $0.92(0.43-1.97), 0.822$ & $0.85(0.37-1.94), 0.697$ & $1.2(0.18-8.15), 0.852$ \\
\hline Taking antidiabetes & $0.79(0.25-2.51), 0.689$ & $0.55(0.14-2.19), 0.392$ & $0.49(0.04-6.48), 0.587$ \\
\hline Taking antidepressant & $1.35(0.72-2.53), 0.355$ & $1.5(0.74-3.03), 0.256$ & $0.41(0.06-2.80), 0.365$ \\
\hline Taking anticoagulant & $1.28(0.58-2.82), 0.548$ & $0.78(0.31-1.97), 0.599$ & $0.77(0.08-7.52), 0.823$ \\
\hline Taking aspirin & $1.11(0.68-1.81), 0.677$ & $1.24(0.71-2.16), 0.450$ & $0.51(0.10-2.46), 0.397$ \\
\hline Taking clopidogrel & $0.85(0.41-1.76), 0.657$ & $0.98(0.44-2.2), 0.972$ & $0.08(0.004-1.79), 0.112$ \\
\hline Having medical insurance & $0.82(0.68-0.99), 0.044^{*}$ & $0.77(0.62-0.96), 0.018^{*}$ & $0.89(0.54-1.46), 0.646$ \\
\hline Employment status (employed vs. unemployed and retired) & $1.32(0.92-1.88), 0.132$ & $1.22(0.79-1.89), 0.371$ & $1.16(0.37-3.66), 0.805$ \\
\hline Education status (educated vs. low education) & $1.02(0.78-1.32), 0.908$ & $1.03(0.76-1.42), 0.834$ & $0.21(0.05-0.81), 0.024^{*}$ \\
\hline Marital status (married vs. single and widowed/divorced) & $1.17(0.76-1.79), 0.476$ & $1.15(0.69-1.89), 0.589$ & $2.04(0.74-5.59), 0.166$ \\
\hline Income (good income vs. $<500,000$ L.L.) & $0.84(0.67-1.06), 0.150$ & $0.91(0.69-1.18), 0.464$ & $1.11(0.58-2.15), 0.752$ \\
\hline
\end{tabular}

*Statistically significant; cardiac diseases include cardiac arrhythmia/atrial fibrillation, coronary heart disease/myocardial infarction, peripheral artery disease, and/or heart failure; In this multivariate analysis, all socioeconomic, demographic, vascular, and behavioral factors were controlled except for cigarette and waterpipe smoking. For total stroke, all stroke cases, ischemic and hemorrhagic, were included in the analysis and compared to all stroke-free patients. For ischemic stroke, all ischemic stroke patients were compared to all stroke-free patients and for hemorrhagic stroke, all hemorrhagic stroke patients were compared to all stroke-free patients, separately; In the multivariate analysis, the -2 log likelihood was 565.295 and Nagelkerke $R^{2}$ was 0.416 for total stroke; the $-2 \log$ likelihood was 439.739 and Nagelkerke $R^{2}$ was 0.426 for ischemic stroke; the -2 log likelihood was 106.724 and Nagelkerke $R^{2}$ was 0.579 for hemorrhagic stroke; OR, odds ratio; CI, confidence interval.

(95\% CI, 1.33-13.59) (Table 4). The risk of total and ischemic strokes was also found to significantly increase among current waterpipe smokers; OR $=4.99$ (95\% CI, 2.07-11.99) and 6.25 (95\% CI, 2.26-17.39), respectively (Table 4).

The association between cigarette and waterpipe smoking and the risk of stroke also depended on the presence of other potential stroke risk factors in individuals, including age, hypertension history, cardiac diseases history, overweight and obesity history, physical activity, medication, and medical insurance (Table 4).

\subsubsection{Dose-effect relationship between smoking and stroke}

The risk of stroke was found to be statistically related to the number of cigarettes smoked. Among current cigarette smokers, for total stroke, the risk significantly increased with an increase in the number of cigarette smoked, OR $=2.26(95 \%$ CI, $1.25-5.25)$ and 3.81 (95\% CI, 1.6-9.01), for 20-60 and $>60$ pack-years, respectively, and similarly for ischemic stroke, 
Table 5 Logistic regression: cigarette dose-effect relationship

\begin{tabular}{|c|c|c|c|}
\hline \multirow{2}{*}{ Major independent variables } & OR adjusted $(95 \% \mathrm{CI}), p$ & OR adjusted $(95 \% \mathrm{CI}), p$ & OR adjusted $(95 \% \mathrm{CI}), p$ \\
\hline & Total stroke $(N=205)$ & Ischemic stroke $(N=143)$ & Hemorrhagic stroke $(N=31)$ \\
\hline \multicolumn{4}{|c|}{ Current cigarette smoker vs. never smoker $(<0.1)$ (pack-years) } \\
\hline$<20$ & 2.41 (0.89-6.47), 0.081 & $2.97(0.88-9.97), 0.079$ & $3.74(0.28-50.0), 0.320$ \\
\hline $20-60$ & $2.26(1.25-5.29), 0.010^{*}$ & $2.40(1.01-5.74), 0.049^{*}$ & $11.52(1.18-112.16), 0.035^{\star}$ \\
\hline$>60$ & $3.81(1.6-9.01), 0.003^{*}$ & $4.37(1.69-11.28), 0.002^{*}$ & $13.30(0.465-380.89), 0.131$ \\
\hline \multicolumn{4}{|c|}{ Former cigarette smoker vs. never smoker $(<0.1)$ (pack-years) } \\
\hline$<25$ & $2.88(0.25-33.45), 0.398$ &,- 0.999 &,- 1.000 \\
\hline $25-36$ & $3.34(0.27-41.71), 0.349$ & $2.11(0.11-40.3), 0.619$ &,- 1.000 \\
\hline$>36$ & $5.75(0.52-63.44), 0.153$ & $3.53(0.31-39.73), 0.307$ &,- 1.000 \\
\hline \multicolumn{4}{|l|}{ Passive cigarette smoking } \\
\hline Exposed to one person smoker & $3.07(1.66-5.67),<0.001^{*}$ & $3.64(1.75-7.58), 0.001^{\star}$ & 5.05 (0.89-28.58), 0.067 \\
\hline Exposed to more than one person smoker & $1.48(0.52-4.25), 0.462$ & $1.37(0.42-0.49), 0.608$ & $0.58(0.008-44.02), 0.807$ \\
\hline
\end{tabular}

*Statistically significant; For passive cigarette smoking, only nonsmokers were included in the analysis; Clarification for the logistic model for current cigarette smoker vs. never smoker: only current cigarette smokers were compared to never smokers (of both, cigarette and waterpipe) based on the pack-years. The dependent variable was total stroke/ischemic stroke/ hemorrhagic stroke. Variables included in the multivariate analysis were: age, sex, education status, marital status, monthly income, employment, insurance, antihypertensive, lipidlowering medication, anticoagulant, antidiabetes, aspirin, clopidogrel, antidepressant, hypertension, cardiac diseases, deep venous thrombosis/pulmonary embolism, diabetes mellitus, dyslipidemia, overweight and obesity, physical activity, cigarette smoking, and waterpipe smoking. Variables controlled in the analysis were: age, sex, education status, marital status, monthly income, employment, insurance, antihypertensive, lipid-lowering medication, anticoagulant, antidiabetes, aspirin, clopidogrel, antidepressant, hypertension, cardiac diseases, deep venous thrombosis/pulmonary embolism, diabetes mellitus, dyslipidemia, overweight and obesity, physical activity, and waterpipe smoking; Clarification for the logistic model for former cigarette smoker vs. never smoker: only former cigarette smokers were compared to never smokers (of both, cigarette and waterpipe) based on the pack-years; CI, confidence interval; OR, odds ratio.

$\mathrm{OR}=2.4(95 \% \mathrm{CI}, 1.01-5.74)$ and 4.37 (95\% CI, 1.69-11.28), for 20-60 and >60 pack-years, respectively (Table 5). Moreover, the hemorrhagic stroke risk significantly increased among current smokers of 20-60 pack-years. Passive cigarette smokers were also at increased risk of total and ischemic stroke with OR $=3.07(95 \%$ CI, 1.66-5.67) and 3.64 (95\% CI, 1.75-7.58), respectively (Table 5).

Smoking $<5$ waterpipe-years among current waterpipe smokers increased the risk of total and ischemic strokes, $\mathrm{OR}=13.16$ (95\% CI, 2.9-59.63) and 15.41 (95\% CI, 2.84-83.71), respectively. An increase in the ischemic stroke risk was also found with $>15$ waterpipe-years among current waterpipe smokers. The risk of hemorrhagic stroke significantly increased with 5-15 waterpipe-years, OR $=40.33$ (95\% CI, 1.09-1499.13). Being a former waterpipe smoker of 10-20 waterpipe-years was also at a significantly increased risk of total and ischemic strokes, $\mathrm{OR}=27.64$ (95\% CI, 1.7-448.21) and 27.93 (95\% CI, 1.33-586.62), respectively. The risk of stroke was also correlated with the duration and frequency of waterpipe smoking (Table 6).

\subsubsection{Stratification by cigarette smoking status}

In an investigation on whether waterpipe smoking is related to stroke, cigarette smoking was suspected of being a confounding factor. Cigarette smoking is an important risk factor of stroke, and is commonly associated with waterpipe smoking. We can eliminate the confounding effect of cigarette smoking by stratifying on this factor: cigarette smokers and non-cigarette smokers.

Table 7 shows that waterpipe smoking has a stronger association with stroke among current cigarette smokers than non-cigarette smokers, OR = 10.34 (95\% CI, 1.43-74.54) and 4.35 (95\% CI, 1.5412.27), respectively.
We also conducted the Breslow-Day test of homogeneity to test the hypothesis that the OR between waterpipe smoking and stroke is the same at each level of cigarette smoking (cigarette smokers and non-cigarette smokers). The $p$-value for Breslow-Day test was 0.562 indicating that we have to accept the hypothesis test (Table 8).

\subsubsection{Stratification by age}

The relationship between smoking and stroke may be confounded by age. Therefore, we stratified on this factor as well: Age $(<65$ years) and ( $\geq 65$ years).

Table 9 showed that current cigarette smoking and waterpipe smoking has a significant association with stroke among patients $\geq 65$ years of age than patients $<65$ years of age, OR $=3.65$ (95\% CI, $1.87-7.12)$ and $\mathrm{OR}=54.03(4.37-668.18)$, respectively.

\section{DISCUSSION}

This study shows that smoking increases the risk of stroke in Lebanon, reaching more than six times the risk of non-smoking.

The majority of our subjects were female (mean age of 63 years), which might explain why they were unemployed, had low education, were married, and earned little income.

Being a current or former cigarette smoker increases the risk of developing a stroke [15]. In Lebanon, stroke-free cigarette smokers were at high risk of developing stroke symptoms [16]. Similarly, in the multivariate analysis of this study and after regulation for confounding (socioeconomic, demographic, and other medical and behavioral history risk factors), being a cigarette smoker increases the risk of developing ischemic and hemorrhagic stroke 
Table 6 Logistic regression: waterpipe dose-effect relationship

\begin{tabular}{|c|c|c|c|}
\hline \multirow{2}{*}{ Major independent variables } & OR adjusted $(95 \% \mathrm{CI}), p$ & OR adjusted $(95 \% \mathrm{CI}), p$ & OR adjusted $(95 \% \mathrm{CI}), p$ \\
\hline & Total stroke $(N=205)$ & Ischemic stroke $(N=143)$ & Hemorrhagic stroke $(N=31)$ \\
\hline \multicolumn{4}{|c|}{$\begin{array}{l}\text { Current waterpipe smoker vs. never smoker (0.1) } \\
\text { (waterpipe-years) }\end{array}$} \\
\hline$<5$ & $13.16(2.9-59.63), 0.001^{*}$ & $15.41(2.84-83.71), 0.002^{*}$ &,- 0.992 \\
\hline $5-15$ & $1.57(0.34-7.19), 0.565$ & $1.33(0.19-9.23), 0.775$ & $40.33(1.09-1499.13), 0.045^{\star}$ \\
\hline$>15$ & $6.31(0.83-48.02), 0.075$ & $12.52(1.55-101.3), 0.018^{\star}$ &,- 0.999 \\
\hline \multicolumn{4}{|c|}{$\begin{array}{l}\text { Former waterpipe smoker vs. never smoker (0.1) } \\
\text { (waterpipe-years) }\end{array}$} \\
\hline$<10$ &,- 0.999 &,- 1.000 &,- 1.000 \\
\hline $10-20$ & $27.64(1.7-448.21), 0.020^{*}$ & $27.93(1.33-586.62), 0.032^{*}$ &,- 1.000 \\
\hline$>25$ &,- 1.000 &,- 1.000 &,- 1.000 \\
\hline \multicolumn{4}{|l|}{ Current waterpipe duration (years) } \\
\hline$<3$ & $8.39(1.87-37.78), 0.006^{*}$ & $7.95(1.76-35.85), 0.007^{\star}$ & $7.86(1.75-35.21), 0.007^{\star}$ \\
\hline \multicolumn{4}{|c|}{ Current waterpipe frequency (waterpipe/day) } \\
\hline$<2$ & 7.48 (2.29-24.37), $0.001^{\star}$ & $7.18(2.27-22.69), 0.001^{*}$ & $6.74(2.10-21.55), 0.001^{*}$ \\
\hline \multicolumn{4}{|c|}{ Former waterpipe duration (years) } \\
\hline$\leq 10$ & $21.84(1.69-281.15), 0.018^{*}$ & $16.15(1.26-206.74), 0.032^{*}$ & $19.27(1.51-245.12), 0.023^{*}$ \\
\hline \multicolumn{4}{|c|}{ Former waterpipe frequency (waterpipe/day) } \\
\hline$<2$ & - & $5.98(1.79-19.87), 0.004^{*}$ & - \\
\hline
\end{tabular}

\footnotetext{
*Statistically significant; Clarification for the logistic model for current waterpipe smoker vs. never smoker: only current waterpipe smokers were compared to never smokers (of both, cigarette and waterpipe) based on the waterpipe-years. The dependent variable was total stroke/ischemic stroke/hemorrhagic stroke. Variables included in the multivariate analysis were: age, sex, education status, marital status, monthly income, employment, insurance, antihypertensive, lipid-lowering medication, anticoagulant, antidiabetes, aspirin, clopidogrel,

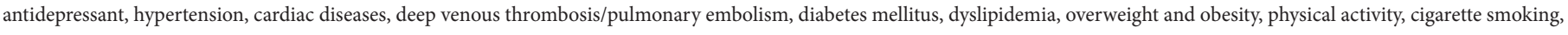
and waterpipe smoking. Variables controlled in the analysis were: age, sex, education status, marital status, monthly income, employment, insurance, antihypertensive, lipid-lowering medication, anticoagulant, antidiabetes, aspirin, clopidogrel, antidepressant, hypertension, cardiac diseases, deep venous thrombosis/pulmonary embolism, diabetes mellitus, dyslipidemia, overweight and obesity, physical activity, and cigarette smoking; Clarification for the logistic model for former waterpipe smoker vs. never smoker: only former waterpipe smokers were compared to never smokers (of both, cigarette and waterpipe) based on the waterpipe-years; CI, confidence interval; OR, odds ratio.
}

Table 7 Adjusted odds ratios for total stroke associated with waterpipe smoking among current cigarette smokers and noncigarette smokers

\begin{tabular}{|c|c|c|c|c|}
\hline \multirow{2}{*}{ Independent variables } & \multicolumn{2}{|c|}{ Cigarette smokers $(N=194)$} & \multicolumn{2}{|c|}{ Noncigarette smokers $(N=410)$} \\
\hline & OR adjusted & $95 \% \mathrm{CI}, p$ & OR adjusted & $95 \% \mathrm{CI}, p$ \\
\hline Current waterpipe smoking & 10.34 & $1.43-74.54,0.02^{\star}$ & 4.35 & $1.54-12.27,0.006^{*}$ \\
\hline Age (years, $\geq$ ) & 1.08 & $1.03-1.14,0.002^{*}$ & 1.01 & $0.99-1.04,0.328$ \\
\hline Hypertension history (presence) & - &,- 0.998 & 9.1 & $2.16-38.34,0.003^{*}$ \\
\hline Cardiac diseases history (presence) & 3.21 & $0.91-11.33,0.071$ & 2.46 & $1.12-5.45,0.026^{\star}$ \\
\hline Overweight and obesity history (presence) & 1.76 & $0.82-3.81,0.150$ & 2.55 & $1.48-4.4,0.001^{*}$ \\
\hline Physical activity (physically active) & 0.46 & $0.17-1.21,0.116$ & 0.49 & $0.27-0.91,0.022^{*}$ \\
\hline Taking clopidogrel & 0.15 & $0.02-0.97,0.047^{\star}$ & 1.32 & $0.47-3.72,0.602$ \\
\hline Insurance (presence) & 0.86 & $0.59-1.26,0.442$ & 0.74 & $0.56-0.98,0.033^{\star}$ \\
\hline
\end{tabular}

*Statistically significant; The odds ratio have been adjusted for all socioeconomic and demographic factors, vascular factors and behavioral factors; Variables included in the multivariate analysis were: age, sex, education status, marital status, monthly income, employment, insurance, antihypertensive, lipid-lowering medication, anticoagulant, antidiabetes, aspirin, clopidogrel, antidepressant, hypertension, cardiac diseases, deep venous thrombosis/pulmonary embolism, diabetes mellitus, dyslipidemia, overweight and obesity, physical activity, and waterpipe smoking. The dependent variable was total stroke. Variables controlled in the analysis were: age, sex, education status, marital status, monthly income, employment, insurance, antihypertensive, lipid-lowering medication, anticoagulant, antidiabetes, aspirin, clopidogrel, antidepressant, hypertension, cardiac diseases, deep venous thrombosis/pulmonary embolism, diabetes mellitus, dyslipidemia, overweight and obesity, and physical activity; CI, confidence interval; OR, odds ratio.

Table 8 Test of homogeneity of the odds ratio

\begin{tabular}{lccccccc}
\hline \multirow{2}{*}{ Independent variable } & \multicolumn{2}{c}{ Cigarette smokers } & & \multicolumn{2}{c}{ Noncigarette smokers } & p-Value for Breslow-Day test \\
\cline { 2 - 3 } & OR unadjusted & $\mathbf{9 5 \%}$ CI & & OR unadjusted & 95\% CI & \\
\hline Current waterpipe smoking & 2.08 & $0.57-7.64$ & & 2.22 & $1.1-4.49$ & 0.562 \\
\hline
\end{tabular}

CI, confidence interval; OR, odds ratio.

by two to fourfold compared with non-smokers. Moreover, this cigarette smoking-stroke relationship is affected by the dose of smoking where the higher the number of cigarettes smoked per day, the higher the risk of stroke. This is in occurrence with Bhat et al. [17] study approving the dose-effect relationship.
Former cigarette smoking is not found to be related to stroke in Lebanon. We hypothesize this to be related to the long period of quitting smoking among former smokers (mean = 14.09 years), in contradiction to the study of Zhang et al. [18]. Although many studies have found that smoking cessation significantly reduces 
Table 9 Adjusted odds ratios for total stroke associated with smoking among patients $<65$ years and patients $\geq 65$ years old

\begin{tabular}{|c|c|c|c|c|}
\hline \multirow{2}{*}{ Independent variables } & \multicolumn{2}{|c|}{ Patients $(<65$ years) $N=273$} & \multicolumn{2}{|c|}{ Patients ( $\geq 65$ years) $N=377$} \\
\hline & OR adjusted & $95 \% \mathrm{CI}, p$ & OR adjusted & $95 \% \mathrm{CI}, p$ \\
\hline Current cigarette smoking & 1.45 & $0.61-3.43,0.4$ & 3.65 & $1.87-7.12,<0.001^{\star}$ \\
\hline Current waterpipe smoking & 2.5 & $0.73-8.56,0.146$ & 54.03 & $4.37-668.18,0.002^{\star}$ \\
\hline Hypertension history (presence) & 10.38 & $1.02-105.34,0.048^{*}$ & 28.35 & $4.4-182.12,<0.001^{\star}$ \\
\hline Cardiac diseases history (presence) & 3.34 & $0.86-12.89,0.081$ & 1.68 & $0.84-3.37,0.145$ \\
\hline Overweight and obesity history (presence) & 1.5 & $0.74-3.04,0.267$ & 2.95 & $1.72-5.06,<0.001^{*}$ \\
\hline Physical activity (physically active) & 0.8 & $0.35-1.85,0.601$ & 0.31 & $0.17-0.59,<0.001^{\star}$ \\
\hline Taking antihypertensive & 0.45 & $0.05-4.46,0.496$ & 0.12 & $0.02-0.8,0.028^{\star}$ \\
\hline Insurance (presence) & 0.93 & $0.64-1.34,0.68$ & 0.72 & $0.57-0.92,0.008^{\star}$ \\
\hline
\end{tabular}

*Statistically significant; The odds ratios have been adjusted for socioeconomic and demographic factors, vascular factors and behavioral factors; CI, confidence interval; OR, odds ratio.

stroke risk in many populations, coming back to a low risk of stroke (almost baseline) is highly related to many factors including smoking duration and cessation, vascular risk factors, and others [15].

Waterpipe smoking is confirmed to increase the risk of ischemic stroke even at a low dose of smoking ( $<5$ waterpipe-years) and the risk of hemorrhagic stroke with a dose of 5-15 waterpipe-years. This type of smoking is very prevalent in Lebanon and the Middle East in general and is extending to other countries including Europe and North America at an alarming level [6]. Waterpipe smokers, similarly to cigarette smokers, develop nicotine/tobacco dependence and are at high risk of many diseases including pulmonary and cardiac diseases as well as cancer. The relationship between stroke and waterpipe smoking is not yet explored; however, only one study published in Lebanon reported an association between stroke symptoms and waterpipe among stroke-free people [16].

The risk of stroke among current waterpipe smokers is found to be higher than the risk among current cigarette smokers. This may be mainly due to the longer sessions of waterpipe smoking compared with cigarette smoking, usually $30 \mathrm{~min}$ for waterpipe smoking compared with only $5 \mathrm{~min}$ for cigarette smoking. Interestingly, waterpipe smoking also showed a stronger association with stroke among cigarette smokers compared with non-smokers. The relationship between waterpipe smoking and stroke is affected by age and the intake of clopidogrel medication among cigarette smokers, while it is affected by the presence of many factors including age, hypertension history, cardiac diseases history, overweight and obesity history, physical activity, and medical insurance among non-cigarette smokers. In addition, when patients were stratified by age, the association between current cigarette smoking and waterpipe smoking remained significant with stroke among older patients ( $\geq 65$ years). This may be attributable to a longer period of smoking among older patients. However, this relationship between smoking and stroke is affected by many other risk factors present in individuals, and the large confidence interval found among current waterpipe smoking indicates the necessity of a study involving a larger age group ( $\geq 65$ years) to compare and confirm these results.

Waterpipe smoking is spreading extensively not only in the Middle East but also worldwide. This study did not assess waterpipe addiction such as nicotine need or psychological yearning, but it suggests that any type of smoking (cigarette or waterpipe) is health alarming in Lebanon, particularly waterpipe, because people perceive it as less harmful and addicting than cigarettes. Refraining and quitting smoking are greatly advised. Increased knowledge for the harm consequences on the health of waterpipe smokers is urgently required.

This study has several strengths. (1) It is the first study to establish a waterpipe-stroke connection. (2) It was conducted at five hospitals in different governorates in Lebanon. (3) No misclassification bias existed as CT scan and/or MRI and neurologist examination classified stroke subtypes at hospitals. (4) All stroke patients $\geq 18$ years of age were included. (5) The questionnaire was well-designed to capture many factors that might affect the relationship between smoking and stroke. (6) The survey was easily conducted in Arabic language. (7) Selection bias, recall bias, and observer's bias were all limited by selecting control patients from the same hospitals as cases, blinding patients to the hypothesis of the study, and having a standardized questionnaire for data collection and a precise protocol for data measurements and analysis.

However, this study has also had many limitations. (1) This study is a hospital-based study that might not represent all Lebanese people; however, we improved the representability of the study by including different hospitals from different governorates. (2) This is a self-reported questionnaire in regard to smoking that might have caused a classification bias as patients try to provide a response that is more socially accepted. Moreover, former smokers might have some recall issues in reporting previous smoking history. (3) The large ORs and wide $95 \%$ confidence intervals found, especially in case of waterpipe smoking, indicate that a larger sample size is required to enhance the assessment of the waterpipe smokingstroke relationship.

From the public health point of view, smoking is a concerning risk factor for stroke that is increasing dramatically worldwide. Smoking cessation reduces the stroke risk after some time of quitting smoking; nevertheless, it needs lots of commitments to achieve and maintain. Effective treatments and programs are available to help in smoking cessation, although limited in Lebanon. Moreover, waterpipe smoking is highly prevalent in Lebanon, particularly among its youth and female adults: an issue that is likely to increase public health awareness. This type of smoking is not fully and carefully considered in intervention programs for prevention such as cigarette smoking regardless of its health risks. We strongly recommend banning waterpipe smoking from public spaces and limit minors' access to this type of smoking. Until fruitful intervention programs for waterpipe smoking are established, medical doctors, public health practitioners, and policy makers should have a role in advising people and patients about the harmful consequences of waterpipe smoking. 


\section{CONCLUSION}

In conclusion, waterpipe smoking is associated with stroke among Lebanese patients. Moreover, the association is stronger among cigarette smokers, demonstrating a quantitative interaction between waterpipe and cigarette smoking.

\section{DISCLOSURES}

The authors declare no conflicts of interest.

\section{REFERENCES}

[1] O’Donnell MJ, Chin SL, Rangarajan S, Xavier D, Liu L, Zhang H, et al. Global and regional effects of potentially modifiable risk factors associated with acute stroke in 32 countries (INTERSTROKE): a case-control study. Lancet 2016;388;761-75.

[2] Rodriguez BL, D’Agostino R, Abbott RD, Kagan A, Burchfiel CM, Yano K, et al. Risk of hospitalized stroke in men enrolled in the Honolulu Heart Program and the Framingham Study: a comparison of incidence and risk factor effects. Stroke 2002;33;230-6.

[3] World Health Organization. WHO report on the Global Tobacco Epidemic. Country profile: Lebanon [Internet]. World health organization; 2015 [cited 2016 Oct 24]. Available from: http://www. who.int/tobacco/surveillance/policy/country_profile/lbn.pdf.

[4] Warren CW, Lea V, Lee J, Jones NR, Asma S, McKenna M. Change in tobacco use among 13-15 year olds between 1999 and 2008: findings from the Global Youth Tobacco Survey. Glob Health Promot 2009;16;38-90.

[5] Akl EA, Gunukula SK, Aleem S, Obeid R, Jaoude PA, Honeine $\mathrm{R}$, et al. The prevalence of waterpipe tobacco smoking among the general and specific populations: a systematic review. BMC Public Health 2011;11;244.

[6] Salameh P, Khayat G, Waked M. Waterpipe smoking in Lebanese women: a lower prevalence but a higher risk of dependence. Eur Respir J 2011;38;4213.

[7] Waked M, Salameh P, Aoun Z. Water-pipe [narguile] smokers in Lebanon: a pilot study [Internet]. 2009 [cited 2017 Feb 6]. Available from: http://apps.who.int/iris/handle/10665/117656.
[8] Zoffice for Human Research Protections. The Belmont Report. 1979 [cited 2016 Oct 24]. Available from: https://www.hhs.gov/ ohrp/regulations-and-policy/belmont-report/.

[9] The World Health Organization MONICA Project (monitoring trends and determinants in cardiovascular disease): a major international collaboration. WHO MONICA Project Principal Investigators. J Clin Epidemiol 1988;41;105-14.

[10] American Stroke Association. Together to End Stroke [Internet]. American Stroke Association; 2013 [cited 2016 May 29]. Available from: http://www.strokeassociation.org/STROKEORG/About Stroke/TypesofStroke/Types-of-Stroke_UCM_308531_SubHomePage.jsp.

[11] El-Hajj M, Salameh P, Rachidi S, Al-Hajje A, Lahoud N, Hassan H. Stroke risk factors: a hospital-based case-control study in lebanon. J R Soc Med Open [Internet] 2017;8;1-10.

[12] World Health Organization. Obesity and Overweight [Internet]. World Health Organization; 2015 [cited 2018 Jan 21]. Available from: http://www.who.int/mediacentre/factsheets/fs311/en/.

[13] US Department of Health and Human Services. 2008 physical activity guidelines for Americans. 2008 [cited 2018 Jan 21]. Available from: http://www.health.gov/PAGuidelines.

[14] Moser M. World Health Organization-International Society of Hypertension Guidelines for the Management of Hypertension-do these differ from the U.S. Recommendations? Which guidelines should the practicing physician follow? J Clin Hypertens (Greenwich) 1999;1:48-54.

[15] Shah RS, Cole JW. Smoking and stroke: the more you smoke the more you stroke. Expert Rev Cardiovasc Ther 2010;8; 917-32.

[16] Farah R, Zeidan RK, Chahine MN, Asmar R, Chahine R, Salameh P, et al. Prevalence of stroke symptoms among strokefree residents: first national data from Lebanon. Int J Stroke $2015 ; 10 ; 83-8$.

[17] Bhat VM, Cole JW, Sorkin JD, Wozniak MA, Malarcher AM, Giles WH, et al. Dose-response relationship between cigarette smoking and risk of ischemic stroke in young women. Stroke 2008;39;2439-43.

[18] Zhang X, Shu XO, Yang G, Li HL, Xiang YB, Gao YT, et al. Association of passive smoking by husbands with prevalence of stroke among Chinese women nonsmokers. Am J Epidemiol 2005;161;213-18. 